\title{
EL PAPEL DE LOS
}

ESTUDIOS GENERALES

EN LOS ESTUDIOS

UNIVERSITARIOS

CONTEMPORÁNEOS

Jorge Rodríguez Beruff*

RESUMEN: Después de una breve síntesis histórica sobre el origen y evolución de los Estudios Generales, el autor ofrece un estado de la cuestión en América Latina y propone siete ideas para los Estudios Generales en el futuro.

yose

ABSTRACT: After a brief historical summary of the origin and growth of General Studies, the author informs us about its current position in Latin America and proposes seven ideas for its future.

PALABRAS ClaVE: Estudios Generales, Movimientos de los Estudios Generales, Ortega y Gasset, Robert Hutchins, Latinoamérica.

KEY WORDS: General Studies, Trends in General Studies, Ortega y Gasset, Robert Hutchins, Latin America.

RECEPCIÓN: 9 de noviembre de 2010.

APROBACIÓN: 7 de diciembre de 2010.

* Universidad de Puerto Rico. 
CITAM Derechos Reservados.

La reproducción total o parcial de este artículo se podrá hacer si el ITAM otorga la autorización previamente por escrito. 


\section{EL PAPEL DE LOS ESTUDIOS GENERALES EN LOS ESTUDIOS UNIVERSITARIOS CONTEMPORÁNEOS*}

A Francisco Polanco

\section{Me honra la invitación que me ha} hecho la Pontificia Universidad Católica Madre y Maestra (PUCMM) para ofrecer esta Conferencia Inaugural. La decisión de auspiciar este II Simposio Internacional sobre Educación General es evidencia del compromiso de muchos años de esta institución con los Estudios Generales. No puedo menos que mencionar con cariño y reconocer las aportaciones de Francisco Polanco y sus colaboradores. A este extraordinario dominicano y universitario le dedico esta conferencia. El mejor tributo que le rinden sus colegas de la PUCMM, es su empeño actual por renovarla y fortalecerla. Él tenía gran interés por asistir al Primer Simposio que llevamos a cabo en la Facultad de Estudios Generales, pero, desgraciadamente, la muerte se interpuso.

Decir compromiso con los Estudios Generales es otra manera de decir compromiso con una educación superior de excelencia. La promoción de ese componente del currículo, y de la experiencia educativa que les provee a los estudiantes, es una forma de reafirmar la posición de liderazgo de esta universidad en el campo de la educación superior en la República Dominicana. A veces, se utilizan nombres distintos para designar los Estudios Generales: ciclo básico, tronco común, estudios humanísticos, artes liberales, educación general, requisitos comunes... También, las instituciones universitarias tienen enfoques diversos para

* Segundo Simposio Internacional de Estudios Generales. Pontificia Universidad Católica Madre y Maestra, 27, 28 y 29 de octubre de 2010, Santiago, República Dominicana. 
JORGE RODRÍGUEZ BERUFF

integrar este componente a su currículo. Por encima de diferencias semánticas o de acercamientos diversos, me atrevo a decir que todos los que estamos en este evento compartimos el propósito de promover los Estudios Generales como un componente fundamental de los estudios universitarios subgraduados. Este evento nos provee la oportunidad de profundizar nuestros conocimientos sobre el tema, conocer nuevas teorizaciones, compartir experiencias y propuestas, y formular proyectos colaborativos regionales que enriquezcan a todas nuestras instituciones, y a otras que, estoy seguro, se irán adhiriendo en el futuro a este esfuerzo.

Prefiero usar aquí el concepto de los Estudios Generales más que el de educación general, que se utiliza a menudo como sinónimo, porque el primer concepto nos ubica más claramente en una tradición universitaria que llevó a una transformación del currículo universitario $y$, en algunos casos, a la fundación de Facultades de Estudios Generales; es decir, a lo que aquí llamo el movimiento de los Estudios Generales. Ese concepto, además, nos vincula históricamente con el momento fundacional de la institución universitaria en Europa y con el ideal humanista que se fue afirmando en esas universidades. Sería interesante que en este evento tratáramos de deslindar la filiación de esos conceptos, que posiblemente expresen matices y connotaciones diferentes.

No podemos hacer una historia de los Estudios Generales en nuestra región, pero creo que es importante que hagamos alguna referencia a su origen y desarrollo. He mencionado un movimiento de los Estudios Generales y debo aclarar ese concepto.

Sabemos que la designación Studium Generale surge en el momento mismo del inicio de la universidad europea y es el nombre que va a llevar esa nueva institución en los siglos XII y XIII. Según Olaf Pedersen, en su libro The first universities: Studium Generale and the Origins of University Education in Europe, se designaba Studium Generale a una institución de educación superior cuando alcanzaba reconocimiento internacional (en ese momento, europeo) y combinaba las artes liberales con alguna especialidad (que eran teología, derecho y medicina). La palabra general significaba principalmente que el Studium atraía estudiantes de toda Europa. 
Eventualmente, el concepto de Universitas, cuyo sentido original se refería al conjunto o universo que formaban las corporaciones de estudiantes y profesores (universitas magistrorum et scholarium), desplazó al concepto original. Pero aún en el siglo XVI se continuaba utilizando la designación de Studium Generale para las universidades coloniales en América, como las de Santo Domingo y San Marcos, las cuales se fundaron con ese nombre, en unos casos siguiendo el modelo del Studium Generale de Salamanca y, en otros, el de Alcalá de Henares.

Pero cuando hablamos de un movimiento de los Estudios Generales nos referimos a un fenómeno relativamente contemporáneo, que se gesta en el siglo XX, a comienzos de la década del 30, como una propuesta de reforma de las universidades estadounidenses y europeas, y que tuvo importantes repercusiones en América Latina. Aunque hay antecedentes para este movimiento en los cambios curriculares que se habían producido en la Universidad de Minnesota y la de Columbia en Estados Unidos, y en movimientos de reforma en América Latina, los eventos más significativos, y casi simultáneos, fueron la publicación de La rebelión de las masas y Misión de la universidad de José Ortega y Gasset, en 1930, y la implantación de un nuevo currículo de Estudios Generales a través del College de la Universidad de Chicago. Esa reforma de Chicago la impulsó, a partir de 1931, el joven presidente Robert Hutchins, quien luego publicara, en 1936, su influyente libro The Higher Learning in America.

Carlos Tünnermann ha señalado que, en el caso de la América Latina, "la República [...] no encontró mejor cosa que hacer con la universidad colonial que sustituirla por un esquema importado, el de la universidad francesa, ideado por Napoleón." Esto le imprimió, según este autor, un carácter profesionalizante a las instituciones universitarias. "La concepción universitaria napoleónica se caracteriza por el énfasis profesionalista, la desarticulación de la enseñanza y la sustitución de la Universidad por una suma de escuelas profesionales, así como la separación de la investigación científica, que deja de ser tarea universitaria y pasa a otras instituciones (Academias e Institutos)." Tünnermann cita al intelectual peruano Luis Alberto Sánchez sobre las consecuencias de 
este modelo universitario: "Como nuestras universidades republicanas empezaron por la profesión para arribar a la cultura, tuvimos y tenemos un conjunto de profesionales incultos y antiuniversitarios." He encontrado referencias a planteamientos en favor de los Estudios Generales en Argentina en el siglo XIX, los cuales no prosperaron por las tendencias profesionalizantes prevalecientes.

El liberal peruano Francisco García Calderón, ya había propuesto en 1912, mucho antes que Ortega hiciera un planteamiento similar, aunque con otras connotaciones, la importancia de que la educación respondiera al "average man", al "hombre medio".

La formación del average man, del ciudadano medio, que preocupa a los sajones, es indispensable en nuestras democracias. A la burocracia excesiva, a las profesiones liberales, se opone esta educación concreta, popular.

La Reforma de Córdova de 1918 sacudió los cimientos de las instituciones universitarias latinoamericanas. Ese movimiento puso énfasis en la necesidad de reestructurar el poder interno de las universidades y su relación con el Estado, superando la mediocridad y el dogmatismo, potenciando así su papel en el desarrollo social. Una de las expresiones de ese movimiento fue la fundación de las "universidades populares". He encontrado una referencia a los Estudios Generales en el currículo propuesto para la Universidad Popular José Martí en Cuba, pero no conozco si el concepto de Estudios Generales estuvo vinculado a la creación de universidades populares en otros países de América Latina, como la Universidad Popular González Prada en el Perú. Sería interesante comparar la propuesta educativa del movimiento de las universidades populares que surge en la década de los veinte y el del movimiento de los Estudios Generales de la década siguiente. Es posible que haya algún paralelismo en lo que se refiere a la necesidad de elevar el nivel cultural de los sectores populares para hacer viables los proyectos democráticos. Debemos explorar estos antecedentes de propuestas alternativas a los modelos profesionalizantes en la educación superior, ya que engarzaron con el movimiento de los Estudios Generales a partir de la década de los treinta. 
La reforma que propuso el movimiento de los Estudios Generales se fundamentó en una crítica de la tendencia cientificista y la sobreespecialización profesional prevaleciente en las universidades. En el caso de los Estados Unidos, representó también un rechazo al pragmatismo a la John Dewey, al "vocacionalismo", al "free elective system” (el sistema de electivas libres que había impulsado el presidente Charles William Eliot, de Harvard) y, a lo que sus propulsores consideraban que era la mediocridad de la experiencia universitaria. A pesar de las diferencias con Dewey, ese movimiento volvió a plantear el tema de la relación entre educación y democracia, analizado en 1916 en el libro Democracy and Education de dicho autor. El tema de la democracia también fue destacado, casi simultáneamente, por la Reforma de Córdova en América Latina. Pero en la coyuntura de la década de los treinta, este asunto se abordó, quizás con mayor urgencia y con otros enfoques, por el ascenso de movimientos autoritarios en Europa y el mundo, particularmente el fascismo y el marxismo dogmático, como le llamaba Ortega al estalinismo.

José Ortega y Gasset y Robert Hutchins fueron las principales figuras intelectuales del movimiento de los Estudios Generales, sin menospreciar el papel que jugaron otros pensadores estadounidenses como Stringfellow Barr, Scott Buchanan y Alexander Meiklejohn, o latinoamericanos, como Ángel Quintero Alfaro y Ernesto Mayz Vallenilla.

Entre Ortega y Hutchins hay puntos de coincidencia e importantes divergencias. Ambos comparten un rechazo al predominio de un cientificismo de raigambre positivista, que ponía énfasis en la formación de científicos y relegaba las materias humanísticas a la función de proveer una “cultura general" de valor ornamental, así como a los planes de estudios profesionales altamente especializados, que no dotaban a los estudiantes de una formación cultural, entendida como el sistema vital de las ideas en cada tiempo. Para Ortega, las materias generales habían quedado como un residuo de lo que había sido la universidad medieval, una institución dedicada a la transmisión de la cultura.

La propuesta de Ortega para la reforma universitaria consistió en la necesidad de crear una Facultad de Cultura, que complementara la formación profesional y la investigación científica. De ahí su afirma- 
JORGE RODRÍGUEZ BERUFF

ción de que la universidad no es ciencia, sino que es, además, ciencia. El otro componente de su propuesta tenía que ver con la proyección social de la universidad, que encontró expresión institucional en los programas de extensión. Hay que añadir que, para Ortega, la reforma universitaria tenía la función de crear las condiciones intelectuales para contrarrestar los movimientos autoritarios de la "sociedad de masas" (lo que Mark Mazower ha llamado La Europa negra) y hacer viable la democracia, lo cual requería superar la fragmentación del conocimiento que caracterizaba a la universidad europea moderna. Este planteamiento lo desarroló en La rebelión de las masas. Ortega nunca descartó la función de las universidades en la formación de profesionales ni en la investigación científica, sino que clamó porque la institución recobrase su carácter cultural e intelectual.

Robert Hutchins también propone un currículo de Estudios Generales para contrarrestar el profesionalismo y el cientificismo positivista. Pero su propuesta se ubica en otra tradición institucional. El currículo de Estudios Generales lo impartiría, según él, una institución intermedia entre la escuela y la universidad, que denomina College, y que abarcaría los dos últimos años de la escuela superior y los primeros de los estudios universitarios. El College sería una instancia formativa general y de acceso masivo que proveería al estudiante el legado del pensamiento occidental por medio del estudio de las "grandes obras", o los clásicos. La universidad, propiamente dicha, sería una instancia posterior que se dedicaría a la investigación y a los estudios especializados para aquellos que desearan y pudieran continuar sus estudios superiores.

Hutchins pensaba que la adquisición del legado intelectual de Occidente y el acceso masivo a una instancia de Estudios Generales contribuiría a formar un ciudadano con capacidad de pensamiento autónomo e integrador, es decir, un sujeto democrático. Sin embargo, el pensamiento de Hutchins, en su forma más extrema, llevó a la identificación de los Estudios Generales con el estudio de las "grandes obras" y a plantear que, de su estudio, se derivaría un canon fijo y seguro del conocimiento. Además, adopta un concepto de Occidente marcadamente eurocéntrico y muy a tono con las alianzas transatlánticas que estaban surgiendo 
para hacerle frente al ascenso del fascismo, las cuales cristalizaron plenamente durante la Segunda guerra mundial.

Por esta razón, se planteó el problema de si el estudio de los clásicos era para aprehender los modelos de pensamiento, las formas de inquirir de sus autores o para aprender sus contenidos como conocimientos "acabados". Además, la categoría "occidental" es sumamente problemática.

Creo que no hay por qué descartar la enseñanza de las "grandes obras" en los Estudios Generales, pero como pretextos para provocar la capacidad de pensamiento y la creatividad de los estudiantes, y no como unos contenidos que expresan un conocimiento terminado y seguro, y menos como una progresión del saber en países y regiones determinadas. Precisamente, lo que debemos lograr es que los estudiantes entiendan el carácter cambiante y tentativo de todo conocimiento humanístico y científico, y que no se trata de un corpus sacrosanto de "verdades" que hay que adquirir para ser culto.

Debemos añadir que la categoría de "clásico" tampoco resulta obvia. Por ejemplo, es difícil no percibir una fuerte inclinación eurocéntrica en la lista de obras propuesta por Mortimer Adler y Robert Hutchins, la cual establece una línea de continuidad entre la antigüedad y la cultura estadounidense. En la actualidad, esa lista contiene un solo autor latinoamericano y se trata de un novelista. Como hemos señalado, esta genealogía del pensamiento era cónsona con el proyecto atlanticista vigente en la Segunda guerra mundial y la posguerra.

Por otro lado, en las expresiones más extremas de los impulsores del movimiento, como el Programa Paidiea de Mortimer Adler, se llegó a concebir el programa de los Estudios Generales con un currículo prescrito y fijo, como el modelo para la educación subgraduada, rechazándose radicalmente toda especialización, profesionalización y posibilidad de elección de los estudiantes.

El movimiento de los Estudios Generales llegó a Puerto Rico, tanto por vía de la propuesta de Ortega y Gasset, como del proyecto de reforma curricular que impulsó Robert Hutchins en la Universidad de Chicago. Las propuestas educativas de Ortega eran conocidas en la isla, al 
menos desde principios de los treinta. Antonio S. Pedreira había planteado la necesidad de superar la "cárcel" del especialismo en su importante ensayo Insularismo, publicado en 1934, donde reconoce al pensamiento de Ortega como una de sus fuentes más importantes. El movimiento de reforma de la década de los treinta aspiraba a fortalecer intelectualmente la universidad y darle coherencia a su currículo.

La reforma universitaria que se inició a principios de los cuarenta, en plena Segunda guerra mundial, se llevó a cabo bajo el liderazgo del rector Jaime Benítez, quien estudió su maestría en el ambiente de los cambios promovidos por Hutchins en Chicago, y escribió su tesis sobre la filosofía de Ortega y Gasset. Él se consideraba un discípulo de Ortega. Benítez y sus colaboradores llevaron a cabo una abarcadora reorganización académico-administrativa. Uno de sus componentes claves fue la fundación de una Facultad de Estudios Generales, a cargo de un currículo "básico" común, claramente inspirada en el concepto de la Facultad de Cultura.

Hay que aclarar, como el propio Benítez hizo, que el desarrollo de los Estudios Generales en Puerto Rico no fue un mero calco de modelos estadounidenses o europeos, sino que representó una readaptación a las condiciones de Puerto Rico de las propuestas educativas del movimiento. En ese proceso colaboraron destacados educadores portorriqueños como Ángel Quintero Alfaro, Domingo Marrero, Viola Lugo, el chileno-portorriqueño José Echeverría y Manuel Maldonado Rivera, entre otros, así como también intelectuales españoles exilados, entre los que estaban Francisco Ayala y Sebastián González García. Francisco Ayala, uno de los intelectuales españoles más importantes, prolíficos y, posiblemente, el más longevo del siglo XX, contribuyó al diseño del nuevo curso de Ciencias Sociales y ha dicho que fue Benítez quien llevó a la práctica la idea de la Facultad de Cultura de Ortega.

Ese proyecto sufrió ajustes y cambios desde sus inicios. El único componente que quedó como curso de dos años en la Facultad de Estudios Generales fue el de Humanidades, que estaba estructurado como un panorama de la cultura occidental, desde la antigüedad hasta el siglo XX, muy influido por los cursos estadounidenses de "Western Civilization". Hay que añadir que estas innovaciones se estaban implantando a la 
vez que se ampliaba considerablemente el acceso a la universidad, lo cual ponía mucha presión para el reclutamiento y preparación de un nuevo profesorado.

La fundación de la Facultad de Estudios Generales de la Universidad de Puerto Rico, a partir de 1943, expresó un consenso institucional. Sin embargo, la orientación occidentalista del currículo provocó controversia, ya que a veces se contrapuso a la discusión sobre la realidad portorriqueña. El propio Benítez la consideraba un antídoto al "insularismo", entendido por él como nacionalismo.

En un mundo cada vez más globalizado, una orientación estrechamente eurocéntrica constituye una limitación de nuestros horizontes intelectuales. Las aportaciones que han hecho académicos como Fidel Tubino, en favor de un enfoque intercultural, tienen mucha pertinencia, no sólo para el mundo andino, sino también para nosotros en el Caribe, una región de gran diversidad cultural y lingüística. Por otro lado, los Estudios Generales no pueden darle la espalda a la consideración a nuestras propias aportaciones intelectuales y culturales, ni a la discusión de los acuciantes problemas que nos aquejan, estableciendo una falsa oposición entre lo universal y lo nacional o local.

Aún así, hay que reconocer que el nuevo programa de Estudios Generales fortaleció la experiencia académica en el Recinto de Río Piedras y contribuyó a darle prestigio a la institución. Nuestra Facultad le ha provisto un perfil propio al Recinto de Río Piedras, el cual cuenta con los más altos índices de retención en Puerto Rico. Aunque no se crearon unidades análogas en los otros recintos, se emuló el componente general de Río Piedras, el cual influyó también en el currículo de algunas universidades privadas.

Ángel Quintero Alfaro, uno de los fundadores, definió una lista de diez funciones que cumplía la educación general. Algunas de esas funciones han perdido vigencia. Sin embargo, su concepto de la educación general es aún de gran actualidad.

La reforma más reciente del currículo, contenida en la Certificación 46 del Senado Académico, fue la culminación de una discusión que abarcó una década. Aparte de los cambios que hizo en la distribución de los créditos, fue una valiosa aportación a la reflexión sobre lo que debe 
ser el bachillerato universitario, el perfil al que aspiramos en nuestros egresados y el papel de los Estudios Generales o educación general. Nos sirvió para clarificar el concepto de Estudios Generales que favorecían la Facultad y el Recinto de Río Piedras. Ese concepto pone énfasis en dos ejes fundamentales: un aspecto epistemológico, que tiene que ver con la construcción e integración del conocimiento; y otro que se refiere al desarrollo de competencias, entendidas éstas ampliamente.

A mi juicio, ambos aspectos son indispensables pero, como señala un documento de nuestra Facultad de 1999, es el primero el que distingue los Estudios Generales, su diferencia específica, ya que el desarrollo de competencias es responsabilidad de todos los niveles educativos. Debo mencionar la importante aportación del profesor Rubén Dávila en la formulación del concepto de Estudios Generales que se recogió en la Certificación 46.

El movimiento de los Estudios Generales encontró arraigo en otros países de América Latina, sobre todo en la Cuenca del Caribe. Los escritos de Ortega tuvieron un importante impacto en el mundo de habla hispana. El filósofo visitó la Argentina en 1916, 1928 y 1938. Sus escritos tuvieron una amplísima difusión. Juan Escámez Sánchez ha destacado la importancia que tuvieron los temas educativos en su obra desde sus primeros escritos. El experimento portorriqueño también debió haber servido como modelo en la posguerra. Y, como en el caso dominicano, hubo otras rutas y vínculos institucionales por medio de los cuales incidió el movimiento en la región.

La influencia de Ortega y del Instituto de Humanidades que había creado en Madrid es evidente, por ejemplo, en un evento auspiciado por la Facultad de Educación de la Universidad Nacional Mayor de San Marcos, cuyas actas fueron publicadas en 1951 bajo el título de La universidad en el siglo $X X$. Ese evento congregó a un grupo muy destacado de intelectuales latinoamericanos y de otras partes del mundo. Fernando de Azevedo, de la Universidad de San Pablo, Brasil, resumió uno de los temas principales de ese encuentro de la siguiente manera:

En otras palabras, las Universidades ganarían, aún desde el punto de vista de la cultura puramente intelectual y profesional, si se transformaran en verdaderas instituciones de formación integral. 
El Rector de la Universidad de Costa Rica, Rodrigo Facio Brenes, fundó la Escuela de Estudios Generales en 1957, como parte de la reforma universitaria. Los Estudios Generales se convirtieron en un componente importante de los estudios universitarios en Costa Rica, con otros programas en la Universidad Nacional de Heredia y la Universidad Estatal de Educación a Distancia (UNED). Luego de la caída de Trujillo en la República Dominicana, el movimiento universitario renovador logró la autonomía de la Universidad Autónoma de Santo Domingo (UASD) y promovió la fundación del Colegio Universitario, a cargo de un currículo común. El Colegio Universitario llegó a atender el 30\% de los estudiantes de la UASD, pero fue eliminado porque "resultaba muy costoso". La UASD aún está pagando el costo académico de esa decisión. En este país, la Pontificia Universidad Católica Madre y Maestra (PUCMM) incorporó desde sus inicios un ciclo básico en su currículo. En otras universidades, como la APEC, que posee un Decanato de Estudios Generales, existen unidades a cargo de los Estudios Generales. El componente de educación general es un requisito para la acreditación de todas las instituciones universitarias dominicanas, aunque habría que ver si realmente se cumple esta estipulación en la práctica.

También en Honduras se creó el Centro Universitario de Estudios Generales (CUEG) como parte de la reforma universitaria. El CUEG fue semillero académico para la Universidad Nacional Autónoma de Honduras (UNAH), pero fue disuelto en el 2008. Tenemos la ventaja de tener entre nuestros participantes al Vicerrector de la UNAH, Ernesto Paz, quien nos podrá explicar el proceso en su país y la situación actual.

En Venezuela, el destacado filósofo y educador Ernesto Mayz Vallenilla, inspirado en las ideas educativas de Ortega, fue el propulsor de los Estudios Generales en la Universidad Simón Bolívar. Es interesante que, en el proyecto de dicha Universidad, le acompañó un exiliado español, Segundo Serrano Poncela, a quien Mayz Vallenilla encargó organizar el programa de Estudios Generales. Serrano había enseñado en la Universidad de Puerto Rico y conocía de cerca la reforma universitaria. Otros intelectuales venezolanos, como el filósofo Juan David García Bacca, se vincularon a las iniciativas a favor de los Estudios Generales. 
JORGE RODRÍGUEZ BERUFF

En 1970, se establecieron facultades de Estudios Generales y de Letras y Ciencias en la Pontificia Universidad Católica del Perú. Aún antes, esa institución incorporaba los Estudios Generales en su currículo. La calidad que ese componente le imprimió a la educación universitaria en esa institución, debe haber sido uno de los factores que la convirtió en modelo para otras universidades peruanas. Es importante mencionar que varias otras universidades peruanas tiene programas de Estudios Generales y algunas participaron en el reciente Simposio auspiciado por la Facultad de Estudios Generales-Letras de la PUCP.

En Argentina se estableció, luego de la dictadura, el Ciclo Básico Común en la Universidad de Buenos Aires, en la que participan 3,000 profesores. El Director de este programa, el profesor Jorge Ferronato, no pudo estar con nosotros en este Simposio, pero lo consideramos parte de esta iniciativa. La Universidad del Magdalena en Colombia creó recientemente una Facultad de Estudios Generales y un programa para promover la democratización del acceso a la universidad y mejorar el nivel de los estudiantes. La Universidad de Chile estableció, en 1994, un exitoso Programa de Bachillerato con enfoque interdisciplinario de Estudios Generales. Según explica Fernando Lolas, ésta ha sido una rica experiencia que ha tenido que superar muchas resistencias por parte de una institución con enfoque profesionalizante. El prestigioso Instituto Tecnológico Autónomo de México (ITAM), posee un Departamento Académico de Estudios Generales, representado aquí por su director Carlos McCadden Martínez. Su concepción de los Estudios Generales es la siguiente:

Estudios Generales se entiende como la entidad académica más propiamente orientada a formar en la persona un tipo de cualidad que le permita su desarrollo integral y le capacite, para ubicarse significativa y responsablemente en el mundo, a partir de una trayectoria histórica.

Ya que estamos en una universidad católica, debemos recalcar que, como muchos de ustedes saben, la promoción de la interdisciplinaridad, la formación cultural de los estudiantes y los Estudios Generales están contenidos en la misión de las universidades católicas de la Améri- 
ca Latina desde la década de los sesenta. El documento "Misión de la universidad Católica en América Latina”, de febrero de 1967, que fuera preparado por un grupo de expertos para el Departamento de Educación del CELAM, plantea que la universidad debe promover "un diálogo verdadero de las disciplinas científicas entre sí, y de éstas con la teología, de modo que se integre en una visión convergente." Además, añade un pasaje que suscribimos plenamente y que tiene igual validez para las universidades laicas y públicas.

No puede una Universidad, que pretenda ser tal, reducirse a formar profesionales. Esto la condenaría tarde o temprano a un inmediatismo pragmático y mediocre. La Universidad debe necesariamente ser cultivo serio y desinteresado de la ciencia. Pero no se acaba aquí la misión de la Universidad. Debe ésta también responder a los interrogantes e inquietudes más profundas del hombre y de la sociedad, es decir, debe ser centro elaborador y difusor de auténtica cultura. Esta misión científica y cultural de la Universidad es la que le da su sentido más profundo y funda su autonomía. No significa ésta una separación o un hermetismo sino la responsabilidad y, por lo mismo, el derecho de realizar su vocación iluminadora y creadora, libre de toda atadura. Ser centro elaborador de cultura -y esto debe ser la Universidad- significa ser conciencia viva de la comunidad humana a la cual pertenece.

No puede la Universidad prescindir de este compromiso vital que pertenece a su esencia misma y que es el sentido más profundo de su libertad y de su autonomía.

Con avances y retrocesos, en modalidades diversas, el movimiento de los Estudios Generales tiene plena vigencia hoy en día en la educación superior en América. En Estados Unidos, la tendencia a reducir o eliminar los componentes de Estudios Generales, que llevó a un empobrecimiento de la educación universitaria, se ha revertido o al menos detenido. El embate contra los programas de Estudios Generales, como ocurrió en Puerto Rico, se ha basado en argumentos diversos, como su obsolescencia, la deseabilidad de aumentar la libertad de elección de los estudiantes, la poca utilidad y el costo de ofrecer este componente, la necesidad de acortar el tiempo de estudios y los créditos de las carre- 
ras, la prerrogativa que debía tener cada programa para determinar todos los requisitos de sus estudiantes, y otros. ¡Un documento en Puerto Rico llegó a plantear que había que revisar nuestro esquema curricular porque comenzaba un nuevo milenio!

Sin embargo, un estudio de 2007 de la Universidad de Harvard, titulado "Report of the Task Force on General Education", subrayó la necesidad de una formación más integral de sus estudiantes para comprender las complejidades culturales, políticas y económicas de un mundo globalizado. El documento señala que la educación general es la instancia en que se logra que los estudiantes entiendan cómo todo lo que enseñamos en las artes liberales se relaciona con su vida y el mundo que confrontarán. La educación general es la cara pública de la educación liberal.

La prestigiosa universidad de enfoque tecnológico y científico, Massachusetts Institute of Technology (MIT), implantó un nuevo programa, recomendado por un comité institucional en 1985-1986 para asegurar que sus estudiantes recibían una exposición amplia a las Humanidades, las Artes y las Ciencias Sociales. Ese programa requiere que completen ocho cursos en esos campos. También podemos mencionar el énfasis que pone la Universidad de Princeton en la formación humanística y general de sus estudiantes.

La existencia de programas o Facultades de Estudios Generales, y de componentes de Estudios Generales en los currículos universitarios requieren compromisos o consensos amplios de las instituciones de educación superior sobre su valor y necesidad en la formación de sus estudiantes. Ese compromiso tiene que estar plasmado, explícita o implícitamente, en el perfil del egresado al que aspira la institución, independientemente de la carrera o especialidad que se escoja. Como aprendimos en Puerto Rico, ese compromiso no es eterno o inmutable. Requiere ser revalidado de tiempo en tiempo sobre la base de la aportación que hacemos a la educación universitaria. Por lo tanto, requiere de los que estamos involucrados en los Estudios Generales, una actitud de renovación y actualización constante de nuestros ofrecimientos y prácticas docentes. Los Estudios Generales requieren un aggiornamento continuo. 
La rutina, el dogmatismo educativo y el anquilosamiento pueden ser nuestros peores enemigos. En Puerto Rico, sufrimos de un cierto inmovilismo, que quizás constituyó una reacción defensiva ante los esfuerzos de ciertos sectores por debilitar nuestro componente. Esa reacción es contraproducente y nos impide ver nuevas formas de cumplir nuestra misión, así como reconocer la pertinencia de nuestra aportación a la educación universitaria.

Es fundamental, también, que logremos una claridad conceptual sobre lo que son y lo que no son los Estudios Generales, lo cual no es incompatible con que reconozcamos la diversidad de acercamientos y de énfasis válidos en nuestro campo. Pero, sabemos que no todo lo que se define como "educación general" o "Estudios Generales", a veces para propósitos de acreditación, lo es. No basta con que un curso no sea especializado o sea interdisciplinario para que sea de educación general. Aún tiene validez el planteamiento de Ortega de que no se trata de proveer una "cultura general" de carácter ornamental. Los cursos de Estudios Generales son cursos universitarios y, como tales, deben tener el mismo nivel de exigencia que cualquier otro curso universitario. Los programas de Estudios Generales no son un "aterrizaje suave" en la universidad, ni representan una continuidad con la educación preuniversitaria. Por el contrario, deben marcar una ruptura y significar un reto intelectual para los estudiantes.

Sobre la definición de Estudios Generales, quiero citar al escritor nicaragüense Sergio Ramírez, quien expresó muy bien, en un seminario que ofreció en nuestra Facultad, de lo que se trata este componente:

Hay una vieja teoría liberal que me parece que sigue siendo una amenaza para el pensamiento que, además de crítico y global, tiene que ser creativo, obviamente, siempre creativo. Y esa vieja proposición de que uno tiene que cuidar sus partes porque el todo se cuida solo. Y ello puede llevar a las mayores atrocidades porque uno se ocupa de su especialidad y el mundo anda solo, y el mundo va a tener siempre una armonía y nadie va ser capaz de quitarle la armonía al mundo [...] Como en la película "Los Tiempos Modernos" de Chaplin, si mi papel es estar moviendo una tuerca o cerrando una tuerca, el mundo va a estar perfecto porque yo estoy cumpliendo mi papel. 
JORGE RODRÍGUEZ BERUFF

Precisamente el nombre de "universidad", universitas, "universal" viene a contradecir ese concepto. Las universidades implican que uno tiene que ocuparse del todo. Es decir, desarrollar una especialidad con responsabilidad científica [...] Uno debe ser responsable de su parte, pero nunca descuidar el todo y saber que la manera de ser crítico es mirar hacia el universo. A mí me parece que la mejor atalaya para mirar hacia el universo es la universidad.

También quiero referirme al concepto de Estudios Generales que formulara el educador portorriqueño Claudio Prieto y que comparto plenamente.

Lo característico de la educación general es su enfoque del contenido como proceso antes que como conclusión, su acercamiento a los reclamos de validez con sano escepticismo y con entendimiento del contexto histórico en que estos se dan: en otras palabras, la visión del conocimiento como proceso de creación humana en todos los contextos -literario, filosófico, científico [...] el examen de hipótesis alternas, el entendimiento del proceso de creación, el análisis de las premisas del razonamiento del autor-, éstos son todos elementos del acercamiento al conocimiento como estructura del pensamiento y como cultura que, a mi entender, es de la esencia misma de la educación general.

En este Simposio tendremos la oportunidad de conocer muchos acercamientos y experiencias diversas. Hay algunas ideas sobre este componente que deseo dejar sobre el tapete para su discusión.

1. Los Estudios Generales son un componente del currículo universitario y, como tal, deben tener el mismo, o aún mayor, nivel de exigencia que cualquier otro componente. Constituyen una ruptura con la experiencia educativa anterior, a la vez que se diferencian de los componentes especializados y profesionales del currículo. Los Estudios Generales no están para suplir "deficiencias", ni para compensar las debilidades de la educación escolar. Esto no implica que no se atienda el desarrollo de las competencias y destrezas de los estudiantes, pero esto se debe hacer sin desmedro del carácter universitario de los cursos. 
2. Los cursos de Estudios Generales deben establecer sinergias positivas con los otros componentes del currículo, aportando a la experiencia subgraduada desde su propia particularidad intelectual y académica. Por esto, no se oponen, sino que enriquecen, la formación especializada y profesional.

3. En un contexto en que las instituciones promueven cada vez más los estudios de posgrado y la investigación, los Estudios Generales deben responder a estas tendencias, promoviendo las competencias de investigación de los estudiantes desde su ingreso a la universidad. No podemos seguir concibiendo las experiencias de investigación como algo "que ocurre después."

4. El componente de Estudios Generales puede estar "verticalizado" o ser "transversal" al programa de estudios, pero debemos reflexionar cómo atendemos la experiencia de primer año de los estudiantes. Además, los Estudios Generales no requieren de un currículo inflexible en que los estudiantes no puedan escoger entre opciones. Sin embargo, es imprescindible tener criterios (o desarrollar rúbricas) para distinguir entre cursos de Estudios Generales y los cursos electivos.

5. Los Estudios Generales son susceptibles de diversas formas de organización académico-administrativa. Sin embargo, es necesario encontrar maneras de propiciar el desarrollo de comunidades académicas dialogantes y la formación permanente del profesorado. Además, deben haber mecanismos en la estructura universitaria para que las unidades o programas reciban los recursos que requieren para cumplir con su misión.

6. Como hemos dicho, la aportación de los Estudios Generales a la educación universitaria, su vigencia, depende de su renovación constante. Debemos incorporar los nuevos enfoques teóricos y los debates actuales. Por ejemplo, las aportaciones de Edgar Morin son de gran relevancia para nuestro campo, así como las teorizaciones sobre el desarrollo de competencias, el interculturalismo, la transdiciplinaridad y la interdiscipliaridad, y otros temas. También debemos tener mecanismos para integrar nuevos enfoques y experiencias pedagógicas exitosas a nuestra práctica docente.

7. Los Estudios Generales son fundamentales para la formación del liderazgo democrático que requieren nuestros países. Le deben proveer a nuestros estudiantes una capacidad analítica que los habilite para integrar saberes diversos y entender los problemas contemporáneos en su complejidad. Martha Nussbaum ha destacado recientemente que los Estudios Generales son indispensables para desarrollar las habilidades necesarias 
"para la ciudadanía en una sociedad democrática pluralista en un mundo cada vez más interconectado."

Hace algún tiempo, estuve en este campus de Santiago de los Caba1leros ofreciendo un taller sobre Estudios Generales, con el colega Pedro Subirats. Me llamó la atención que las preocupaciones y los debates eran muy similares a los que tenemos en Puerto Rico. Esa sensación la tuve recientemente en el Simposio de la Universidad Católica del Perú y en otras ocasiones cuando he compartido con colegas de diversos países que se desempeñan en este campo.

Es indispensable promover la comunicación y la colaboración entre nosotros. Este evento constituye una instancia de un diálogo necesario. Se trata de un nuevo diálogo regional sobre los Estudios Generales, el cual se ha ido ampliando gradualmente para conformar lo que llamaría el filósofo chileno Eduardo Devés, una red intelectual, la cual esperamos que tenga eventualmente alcance continental. En la Declaración de Río Piedras seis instituciones hicimos un llamado a la constitución de una Red Internacional de Facultades y Unidades a cargo de los Estudios Generales. Ese propósito lo reafirmamos en el evento del Perú. Le agradecemos a la PUCMM el generoso esfuerzo que ha hecho en la organización de este evento, que nos permite constatar la eficacia de nuestra convocatoria y nos debe colocar firmemente en la ruta de la red institucional que estamos construyendo. Estoy seguro de que las deliberaciones de los próximos dos días serán de gran provecho para todos nosotros. Aquí estamos retomando, en las circunstancias del siglo XXI, el movimiento de los Estudios Generales. 